\title{
NUMERICAL STUDY OF THE VERY FORWARD BACKGROUND FROM THE PROTON-PROTON COLLISIONS IN THE EXPERIMENTAL INSERTIONS OF THE LHC
}

\author{
H.Burkhardt ${ }^{1}$, D.Macina ${ }^{2}$, V.Talanov ${ }^{3}$ and E.Tsesmelis ${ }^{2}$
}

\begin{abstract}
The results from the numerical DPMJET-FLUKA simulation of the background in the experimental insertion regions of the LHC are presented. DPMJET3 is used for the generation and analysis of the products from the p-p collision leaving the interaction point in the very forward region. A multiparticle transport code FLUKA is used for the simulation of the resulting secondary cascades in the structure of the LHC Long Straight Sections. The background formation is estimated and analyzed in the LSSs at the locations of the TAN absorbers, Roman Pot stations and Beam Loss Monitors, for the purposes of the machine protection and planning of the operation of the detectors.
\end{abstract}

${ }^{1}$ AB Division, CERN, Geneva, Switzerland

${ }^{2}$ TS Division, CERN, Geneva, Switzerland

${ }^{3}$ TS Division, CERN, Geneva, Switzerland (on leave from IHEP, Protvino, Russia)

Presented at the Twenty Second Particle Accelerator Conference (PAC 2007)

25-29 June 2007, Albuquerque, New Mexico, USA

Administrative Secretariat

LHC Division

CERN

CH - 1211 Geneva 23

Switzerland 


\title{
NUMERICAL STUDY OF THE VERY FORWARD BACKGROUND FROM THE PROTON-PROTON COLLISIONS IN THE EXPERIMENTAL INSERTIONS OF THE LHC
}

\author{
H. Burkhardt, D. Macina, V. Talanov*, E. Tsesmelis, CERN, Geneva, Switzerland
}

\begin{abstract}
The results from the numerical DPMJET-FLUKA simulation of the background in the experimental insertion regions of the LHC are presented. DPMJET3 is used for the generation and analysis of the products from the p-p collision leaving the interaction point in the very forward region. A multi-particle transport code FLUKA is used for the simulation of the resulting secondary cascades in the structure of the LHC Long Straight Sections. The background formation is estimated and analyzed in the LSSs at the locations of the TAN absorbers, Roman Pot stations and Beam Loss Monitors, for the purposes of the machine protection and planning of the operation of the detectors.
\end{abstract}

\section{INTRODUCTION}

The high luminosity experimental insertions IR1 and IR5 of the LHC [1] host several experiments for the purposes of the very forward physics programme. The detectors of these experiments are placed in the dedicated locations along the Long Straight Sections (LSSs) of the machine that are shown in Figure 1. The ATLAS Zero Degree Calorimeters (ZDCs) [2], LHCf experiment [3] and BRAN monitors will be located inside the TAN absorbers each side in IR1 while the TAN absorbers in IR5 will house the CMS ZDCs [4] and another two BRAN monitors as well. The Roman Pot stations of TOTEM [5] XRP1 and XRP3 will be installed in IR5 between the TAN and the D2 separation dipole and in the Q5-Q6 region respectively while the Roman Pots of ATLAS [6] will be located between the Q6 and Q7 quadrupoles of the IR1. Even further in the machine tunnel in the dispersion suppressor region will be installed the detectors of the FP420 experiment [7].

The estimation of the radiation levels and the evaluation of the signal and background at the locations of the detectors are an important issue in the design of the detectors and in the optimization of the detector performance. $\mathrm{Nu}$ merical study of the formation of the particle fluxes in the LSSs that are initiated by the proton-proton collisions in the interaction points of the experimental IRs provides the detailed information on those subjects.

\section{SIMULATION OF THE P-P COLLISIONS}

Secondary cascades initiated by the products from the $\mathrm{p}-\mathrm{p}$ collisions in the interaction points (IPs) that enter the machine tunnel in the very forward direction are the main

\footnotetext{
*Vadim.Talanov@cern.ch
}

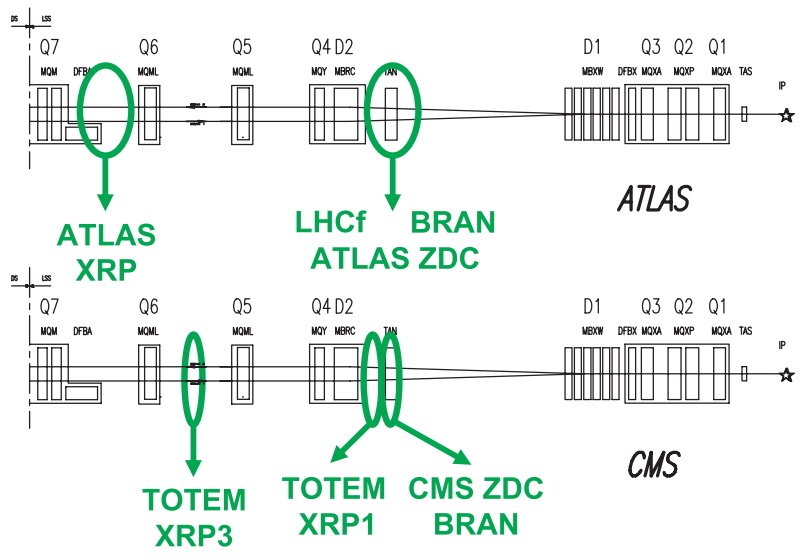

Figure 1: Very forward detectors in the LHC insertions IR1 and IR5 of the ATLAS and CMS experiments.

source of the background in the LSSs of the LHC. To simulate the primary p-p interactions DPMJET v.3.0-4 [8] was used with the default settings of the generator assuming the full crossing angle of $285 \mu \mathrm{rad}$ and the horizontal plane of the beam crossing (IR5 case, studies for IR1 are in progress) for the collisions at nominal energy of $7 \mathrm{TeV}$ and also at $450 \mathrm{GeV}$ at injection. Simulations were done for the full angular range of the produced primary particles and with the cut of $0.89 \mathrm{mrad}$ on the $\theta$ angle of the outgoing particle track $(|\eta|>7.8)$ that is defined by the aperture of the TAS absorber between the interaction point and the machine tunnel.

Average number of particles and total particle energy calculated with and without the cut on the pseudorapidity for different types of particles produced in one p-p event

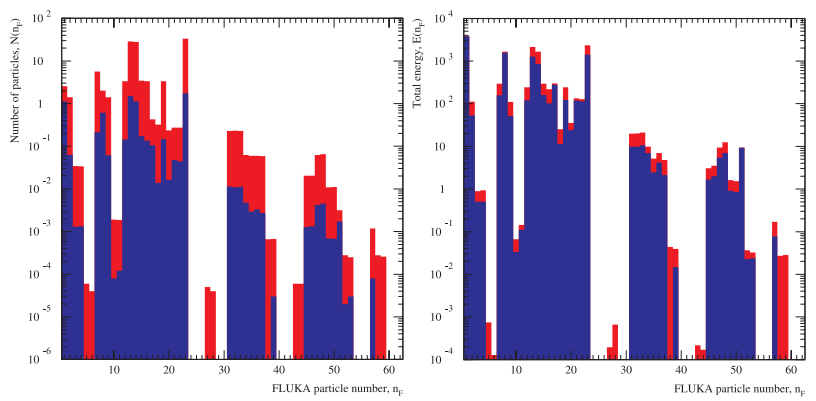

Figure 2: Number of particles (left) and total particle energy, [GeV] (right) per $1 \mathrm{p}$-p event at the $7 \mathrm{TeV}$ beam energy, for the different particle types in the FLUKA coding scheme, with (blue) and without (red) pseudorapidity cut. 


\begin{tabular}{|crrrrrr|}
\hline Symbol & ID & $\mathbf{N}$ & $\mathbf{E}$ & $\mathbf{N} / \mathbf{N}_{0}$ & $\mathbf{E} / \mathbf{N}_{0}$ & $\mathbf{E} / \mathbf{N}$ \\
\hline \hline$p$ & 1 & 1439 & $2.18 \times 10^{6}$ & $7.20 \times 10^{-3}$ & 10.88 & 1511.51 \\
$\bar{p}$ & 2 & 448 & $9.52 \times 10^{4}$ & $2.24 \times 10^{-3}$ & 0.48 & 212.53 \\
$e^{-}$ & 3 & 120 & $3.52 \times 10^{3}$ & $6.00 \times 10^{-4}$ & 0.02 & 29.36 \\
$e^{+}$ & 4 & 50 & 8.9 & $2.50 \times 10^{-4}$ & $5 \times 10^{-5}$ & 0.18 \\
$\gamma$ & 7 & 119630 & $8.72 \times 10^{7}$ & $5.98 \times 10^{-1}$ & 436.24 & 729.32 \\
$n$ & 8 & 46055 & $1.33 \times 10^{8}$ & $2.30 \times 10^{-1}$ & 663.35 & 2880.69 \\
$\bar{n}$ & 9 & 3172 & $2.94 \times 10^{6}$ & $1.59 \times 10^{-2}$ & 14.70 & 926.97 \\
$\mu^{+}$ & 10 & 3 & $2.64 \times 10^{2}$ & $1.50 \times 10^{-5}$ & 0.00132 & 87.89 \\
$\mu^{-}$ & 11 & 2 & $4.83 \times 10^{1}$ & $1.00 \times 10^{-5}$ & 0.00241 & 24.14 \\
$K^{0}$ & 12 & 5292 & $6.03 \times 10^{6}$ & $2.65 \times 10^{-2}$ & 30.13 & 1138.84 \\
$\pi^{+}$ & 13 & 7323 & $6.01 \times 10^{5}$ & $3.66 \times 10^{-2}$ & 3.01 & 82.08 \\
$\pi^{-}$ & 14 & 7992 & $9.96 \times 10^{5}$ & $4.00 \times 10^{-2}$ & 4.98 & 124.66 \\
$K^{+}$ & 15 & 790 & $5.60 \times 10^{4}$ & $3.95 \times 10^{-3}$ & 0.28 & 70.88 \\
$K^{-}$ & 16 & 637 & $3.01 \times 10^{4}$ & $3.19 \times 10^{-3}$ & 0.15 & 47.25 \\
$\Lambda$ & 17 & 5005 & $1.79 \times 10^{7}$ & $2.50 \times 10^{-2}$ & 89.43 & 3573.60 \\
$\bar{\Lambda}^{-}$ & 18 & 182 & $3.03 \times 10^{5}$ & $9.10 \times 10^{-4}$ & 1.51 & 1664.03 \\
$K_{S}^{0}$ & 19 & 607 & $1.29 \times 10^{6}$ & $3.04 \times 10^{-3}$ & 6.43 & 2117.85 \\
$\Sigma^{+}$ & 21 & 1 & $5.99 \times 10^{3}$ & $5.00 \times 10^{-6}$ & 0.03 & 5992.58 \\
$K^{0}$ & 24 & 482 & $7.16 \times 10^{4}$ & $2.41 \times 10^{-3}$ & 0.36 & 148.50 \\
$\bar{K}^{0}$ & 25 & 485 & $9.83 \times 10^{4}$ & $2.43 \times 10^{-3}$ & 0.49 & 202.66 \\
$\Xi^{0}$ & 34 & 66 & $1.82 \times 10^{5}$ & $3.30 \times 10^{-4}$ & 0.91 & 2762.30 \\
$\Xi^{0}$ & 35 & 14 & $2.82 \times 10^{4}$ & $7.00 \times 10^{-5}$ & 0.14 & 2016.03 \\
\hline
\end{tabular}

Table 1: Number of primary particles and their total and mean energy at the TAN central area for $2 \times 10^{5} \mathrm{p}$-p events.

in the positive $z$ direction are shown in Figure 2. The average multiplicity was found to be 120 particles/event at the $7 \mathrm{TeV}$ energy of which only $\sim 7.3$ particles/event pass through the TAS aperture into the tunnel to the left and right parts of the LSS. At the same time the average energy flux with the $\eta$ cut was estimated to be $\sim 10.3 \mathrm{TeV} /$ event which shows that the pseudorapidity cut removes the low-energy component of the signal from the p-p collisions at large $\theta$ that has the highest multiplicity. As can be seen from Figure 2 after the cut $\sim 1$ proton per event (particle type 1) remains with the energy close to the energy of the beam.

The calculated angular distributions are given in Figure 3 for the flux of all particle types and separately for the neutral ones. Vertical line in Figure 3 designates the TAN central area where the sensitive elements of the detectors will be located. Comparison between the angular distributions at $7 \mathrm{TeV}$ and $450 \mathrm{GeV}$ shows that not only the multiplicity of the particles in the p-p event should be expected to be significantly lower in the collisions at the injection energy but also the angular distribution for all particle types is much broader in this case. The last observation makes the detection of the forward neutral particles from the p-p collisions at $450 \mathrm{GeV}$ even more difficult than at $7 \mathrm{TeV}$.
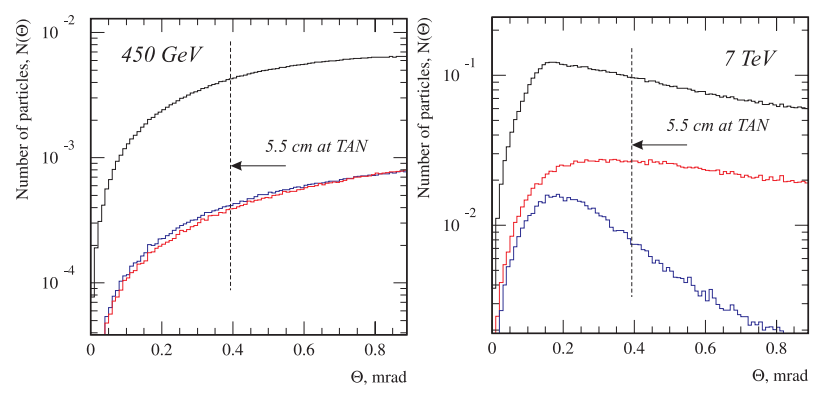

Figure 3: Particle angular distribution, $\mathrm{N}(\theta)$ per $1 \mathrm{p}$-p event at the $450 \mathrm{GeV}$ (left) and $7 \mathrm{TeV}$ (right) beam energy, for all particle types (black), $\pi^{0}$ and $\gamma$ (red), and neutrons (blue).

\begin{tabular}{|crrrrrr|}
\hline Symbol & ID & $\mathbf{N}$ & $\mathbf{E}$ & $\mathbf{N} / \mathbf{N}_{0}$ & $\mathbf{E} / \mathbf{N}_{0}$ & $\mathbf{E} / \mathbf{N}$ \\
\hline \hline$p$ & 1 & 34176 & $3.00 \times 10^{6}$ & $1.71 \times 10^{-1}$ & 15.02 & 87.90 \\
$\bar{p}$ & 2 & 3940 & $2.40 \times 10^{5}$ & $1.97 \times 10^{-2}$ & 1.20 & 61.03 \\
$e^{-}$ & 3 & 1902420 & $3.69 \times 10^{5}$ & 9.512 & 1.84 & 0.19 \\
$e^{+}$ & 4 & 1772620 & $3.72 \times 10^{5}$ & 8.863 & 1.86 & 0.21 \\
$\gamma$ & 7 & 5722660 & $9.14 \times 10^{7}$ & 28.61 & 456.77 & 15.96 \\
$n$ & 8 & 212082 & $1.34 \times 10^{8}$ & 1.06 & 671.80 & 633.53 \\
$\bar{n}$ & 9 & 6634 & $3.15 \times 10^{6}$ & $3.32 \times 10^{-2}$ & 15.77 & 475.30 \\
$\mu^{+}$ & 10 & 1534 & $1.02 \times 10^{4}$ & $7.67 \times 10^{-3}$ & 0.05 & 6.64 \\
$\mu^{-}$ & 11 & 1545 & $9.48 \times 10^{3}$ & $7.73 \times 10^{-3}$ & 0.05 & 6.13 \\
$K^{0}$ & 12 & 5413 & $6.03 \times 10^{6}$ & $2.71 \times 10^{-2}$ & 30.14 & 1113.71 \\
$\pi^{+}$ & 13 & 77274 & $1.43 \times 10^{6}$ & $3.86 \times 10^{-1}$ & 7.16 & 18.53 \\
$\pi^{-}$ & 14 & 79570 & $1.91 \times 10^{6}$ & $3.98 \times 10^{-1}$ & 9.56 & 24.03 \\
$K^{+}$ & 15 & 8542 & $1.83 \times 10^{5}$ & $4.27 \times 10^{-2}$ & 0.91 & 21.39 \\
$K^{-}$ & 16 & 6578 & $1.34 \times 10^{5}$ & $3.29 \times 10^{-2}$ & 0.67 & 20.39 \\
$\Lambda$ & 17 & 5175 & $1.79 \times 10^{7}$ & $2.59 \times 10^{-2}$ & 89.53 & 3460.24 \\
$\bar{\Lambda}$ & 18 & 230 & $3.06 \times 10^{5}$ & $1.15 \times 10^{-3}$ & 1.53 & 1331.71 \\
$K^{0}$ & 19 & 609 & $1.29 \times 10^{6}$ & $3.04 \times 10^{-3}$ & 6.43 & 2111.03 \\
$\Sigma^{-}$ & 20 & 3 & $8.55 \times 10^{1}$ & $1.50 \times 10^{-5}$ & 0.0004 & 28.49 \\
$\Sigma^{+}$ & 21 & 1 & $5.99 \times 10^{3}$ & $5.00 \times 10^{-6}$ & 0.03 & 5992.58 \\
$K^{0}$ & 24 & 4099 & $1.32 \times 10^{5}$ & $2.05 \times 10^{-2}$ & 0.66 & 32.09 \\
$\bar{K}^{0}$ & 25 & 4104 & $1.63 \times 10^{5}$ & $2.05 \times 10^{-2}$ & 0.81 & 39.68 \\
$\bar{\Sigma}^{+}$ & 33 & 2 & $6.64 \times 10^{1}$ & $1.00 \times 10^{-5}$ & 0.0003 & 33.21 \\
$\Xi^{0}$ & 34 & 73 & $1.83 \times 10^{5}$ & $3.65 \times 10^{-4}$ & 0.91 & 2500.70 \\
$\bar{\Xi}^{0}$ & 35 & 16 & $2.83 \times 10^{4}$ & $8.00 \times 10^{-5}$ & 0.14 & 1766.19 \\
$\Xi^{+}$ & 37 & 1 & $3.79 \times 10^{1}$ & $5.00 \times 10^{-6}$ & 0.0002 & 37.94 \\
\hline
\end{tabular}

Table 2: Total number of particles and their total and mean energy at the TAN central area for $2 \times 10^{5} \mathrm{p}$-p events.

\section{PARTICLE FLUX AT THE TAN IN IR5}

Transport of the particles that pass through the TAS absorber into the LSS was performed for the LHC collision optics version 6.5 and the latest layout of the right side of the LSS5 with the FLUKA 2006.3b program [9]. This choice of the simulation code was stipulated by the necessity to model correctly both transport and interactions of the large variety of particle types that can be seen at the distributions of the secondaries from the p-p event at $7 \mathrm{TeV}$ in Figure 2 and to enable the future analysis of the signal per event in the IP or per bunch crossing.

An overview of the calculated particle distributions at the TAN central area of $5.5 \times 5.5 \mathrm{~cm}^{2}$ at $139.8 \mathrm{~m}$ from IP5 in the right part of LSS5 is shown in Tables 1 and 2. The total number of particles $\mathrm{N}$, their total energy $\mathrm{E}[\mathrm{GeV}]$, average number of particles per $\mathrm{p}-\mathrm{p}$ event $\mathrm{N} / \mathrm{N}_{0}$, average energy per event $\mathrm{E} / \mathrm{N}_{0}$ and mean energy $\mathrm{E} / \mathrm{N}$ (both in $\mathrm{GeV}$ ) for all particle types considered in the simulations are given for $\mathrm{N}_{0}=2 \times 10^{5} \mathrm{p}$-p events at the IP.

Table 1 gives these quantities for the primary particles that are seen at the TAN directly from the IP. There the flux of the energy in the TAN central area is completely dominated by the primary neutrons and $\gamma$ 's with the visible contribution from $\Lambda$ 's. The particles of these types have a mean energy close to or a few $\mathrm{TeV}$ and clearly define the "neutral" signal from the point of the p-p collision.

In addition to Table 1, Table 2 gives the same quantities for the total particle flux in which together with the primary particles the products from the simulated secondary cascades are included. As can be seen, an enormous fraction of the secondary $\gamma$ 's ( $\sim 29$ per event compared to $\sim 0.6$ directly from the IP) do not contribute much to the total energy of these particles since their mean energy is more than $\sim 50$ times lower than the energy of the primaries. The same is true for the flux of the secondary neutrons 


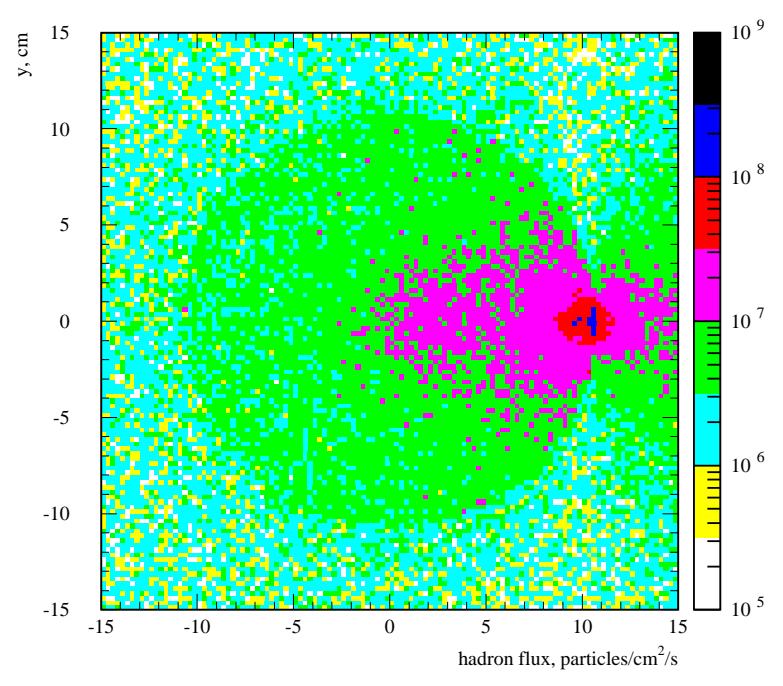

Figure 4: Hadron flux density, [particles $/ \mathrm{cm}^{2} / \mathrm{s}$ ] at the front surface of the TAN absorber in IR5, given for the rate of $8 \times 10^{8}$ p-p inelastic interactions/s in the IP.

and for the background charged hadrons as well although the contribution to the total energy flux in the TAN central area from the last component that is swept by the magnetic structure of the LSS is of the order of a few percent.

The distribution of the simulated secondary particle flux at the TAN front surface is illustrated by Figure 4 . The peak hadron flux density is observed in the TAN outer aperture and the general view of the distribution reflects both the magnetic structure of the LSS and the shape of the recombination chamber in the D1-D2 region. For presentation reasons this distribution was normalized per $8 \times 10^{8} \mathrm{p}$-p interactions/s which implies the use of the $80 \mathrm{mb}$ for the cross-section of the p-p inelastic interaction at $7 \mathrm{TeV}$ and the maximal luminosity of $10^{34} \mathrm{~cm}^{-2} \mathrm{~s}^{-1}$ at IP5 to make these results directly comparable with the available similar data from [10].

\section{CONCLUSION}

The obtained results for the analysis of the secondary particle fluxes were compared with the available independent estimates. The estimated power of the particle flux at the TAN central area equals $168 \mathrm{~W}$ for the rate of the $8 \times 10^{8}$ p-p inelastic interactions/s in IP5 that is consistent with the value of $164 \mathrm{~W}$ given in [11]. Calculated particle spectra at the TAN front surface were compared with the results of the simulations in the LHCf experiment and an agreement on both absolute values and the shape of the energy distributions was observed for all particle types [12]. The participating experiments were provided with the recorded files of the signal and background from the described simulations for the further analysis in the collaborations.

\section{REFERENCES}

[1] O. Brüning, P. Collier, P. Lebrun et al. (editors), "LHC Design Report. Volume I The LHC Main Ring”, CERN 2004003, Geneva, 2004.

[2] ATLAS Collaboration, "Zero Degree Calorimeters for ATLAS”, CERN LHCC 2007-001, Geneva, 2007.

[3] LHCf collaboration, "Technical Design Report of the LHCf Experiment: Measurement of Photons and Neutral Pions in the Very Forward Region of the LHC', CERN LHCC 2006004, Geneva, 2006.

[4] O.A. Grachov, B. Metzler, M. Murray et al., "Measuring Photons and Neutrons at Zero Degrees in CMS", Presented at 19th International Conference on Ultra-relativistic Nucleus-nucleus Collisions Quark Matter 2006, Shanghai, China, November 14-20 2006; nucl-ex/0703001, submitted to Int. J. Mod. Phys. E.

[5] The TOTEM Collaboration, "TOTEM Technical design Report: Total Cross Section, Elastic Scattering and Diffraction Dissociation at the Large Hadron Collider at CERN", CERN LHCC 2004-002, Geneva, 2004.

[6] ATLAS Collaboration, "ATLAS Forward Detectors for Luminosity Measurement and Monitoring", CERN LHCC 2004-010, Geneva, 2004.

[7] B. Cox et al., "FP420: An R\&D Proposal to Investigate the Feasibility of Installing Proton Tagging Detectors in the 420 $m$ Region of the LHC", CERN LHCC 2005-025, Geneva, 2005.

[8] S. Roesler, R. Engel, J. Ranft, "The Monte Carlo Event Generator DPMJET-III", In Proceedings of the Monte Carlo 2000 Conference, Lisbon, October 23-26 2000, A. Kling, F. Barao, M. Nakagawa, L. Tavora, P. Vaz (editors), SpringerVerlag Berlin, 1033-1038 (2001).

R. Engel, "Photoproduction within the two-component Dual Parton Model: Amplitudes and Cross Sections", Z. Phys. C66 (1995) 203.

R. Engel and J. Ranft, "Hadronic photon-photon collisions at high energies", Phys. Rev. D54 (1996) 4244.

[9] A. Fasso', A. Ferrari, J. Ranft and P.R. Sala, "FLUKA: a multi-particle transport code", CERN 2005-10 (2005), INFN/TC_05/11, SLAC-R-773.

A. Fasso', A. Ferrari, S. Roesler et al., "The physics models of FLUKA: status and recent developments", Computing in High Energy and Nuclear Physics 2003 Conference (CHEP2003), La Jolla, CA, USA, March 24-28, 2003, (paper MOMT005) eConf C0303241 (2003), arXiv:hep$\mathrm{ph} / 0306267$.

[10] N.V. Mokhov, I.L. Rakhno, J.S. Kerby, J.B. Strait, "Protecting LHC IP1/IP5 Components Against Radiation Resulting from Colliding Beam Interaction", CERN LHC Project Report 633, Geneva, 2003.

[11] N. Mokhov, "MARS15 Studies of Absorbed and Residual Doses in the TAN and LHCf and ZDC Detectors", First TAN Integration Workshop, CERN, March 102006.

[12] V. Talanov, "Signal and Background Simulation at the Recombination Chamber", Joint LHC Machine-Experiments Workshop on Very Forward Detectors, CERN, January 25 2007. 